D. RODRÍGUEZ

F. J. GIL

J. A. PLANELL

\section{Modelo de la lubricación hidrodinámica de la articulación de una prótesis de cadera}

\section{Hydrodynamic lubrication model of a prosthetic hip articulation}

\section{Resumen}

En este trabajo se estiman las presiones a la cual está sometida la articulación de la cadera por los líquidos fisiológicos, esta aproximación se ha realizado basado en las ecuaciones de mecánica de fluidos y considerando la geometría de la prótesis de cadera. Estos datos son de gran importancia para el diseño de nuevas prótesis de cadera y para mejorar la resistencia al desgaste entre la prótesis y el acetábulo. Una vez obtenida la ecuación-modelo de lubricación articular, se han obtenido valores para un caso general de presiones de alrededor de los $120 \mathrm{KPa}$.

Palabras clave: Biotribología. Cadera. Fluidos. Presión.

\section{Summary}

The pressure that physiological fluids exercise on the hip joint was estimated using fluid mechanics equations and the geometry of hip prostheses. This information is important for the design of new hip prostheses and improvement of the resistance to wear of the prosthesis and cup. After the model equation for articular lubrication was obtained, values were calculated for a general case of pressures of about $120 \mathrm{KPa}$.

Key words: Biotribology. Hip. Fluids. Pressure.

\section{Introducción}

La prótesis de cadera es un tipo de implante que permite recuperar la funcionalidad perdida de la articulación de la cadera. En el caso más usual, se sustituye la articulación natural por una unión bola-hemiesfera cóncava, o acetábulo con un encaje más bien perfecto (superficies conformadas).

A diferencia de la articulación natural, que acostumbra a durar más que la vida de la persona, la articulación de una prótesis artificial presenta fuertes evidencias de desgaste pasados pocos años de su implantación al cuerpo. Si bien se ha demostra-

\section{Correspondencia:}

D. RODRÍGUEZ. Dept. Ciencia de los Materiales e Ingeniería Metalúrgia. E.T.S. Ingenieros Industriales de Barcelona. Universidad Politécnica de Cataluña. Av. Diagonal 647. 08028 Barcelona. do la existencia de diferentes tipos de desgaste, el más usual es un desgaste abrasivo por tercera partícula y un desgaste producido por fatiga subsu-
perficial.

A fin de estudiar la posibilidad de que estos últimos tipos de desgaste sean provocados por una presión excesiva en puntos concretos de la articulación, se estudia en este trabajo la distribución de presiones del acetábulo, mediante la incorporación de la teoría de fluidos a la cinemática y dinámica de movimiento de la articulación de la ca-

\section{Cinemática y dinámica de la articulación de cadera}

La articulación de la cadera se puede considerar como un rodamiento hemiesférico conformado. Está construida de forma que permite el amplio 
rango de movimientos necesarios para las actividades diarias.

En la articulación natural la superficie cóncava, o acetábulo, está recubierta de una capa de cartílago que permite amortiguar los picos de presión sobre la articulación. La cabeza femoral es el componente convexo de la articulación. De hecho, no llega a formar una esfera sino nada más que $2 / 3$ partes de la esfera.

Por lo que se refiere a la cinemática de la articulación, el movimiento tiene lugar en tres planos: sagital $\left(140^{\circ}\right)$, frontal $\left(30^{\circ}\right)$ y transversal $\left(90^{\circ}\right)$. Durante el movimiento, las superficies efectúan un desplazamiento de deslizamiento de la bola en el acetábulo y esto supone que el movimiento es tangencial. Respecto a la dinámica del movimiento, el cálculo de valores de las fuerzas ejercidas sobre la articulación muestran que, si bien en una fase deambulatoria el peso soportado por la articulación es de un máximo de cuatro veces el peso corporal, en fase de carrera o de salto, el pico de carga puede llegar a las nueve veces el peso corporal.

\section{Lubricación}

\section{Lubricación de la articulación de cadera}

Los regímenes de lubricación se dividen, según su modo de funcionamiento, en:

- lubricación hidrodinámica: se caracteriza generalmente, por la presencia de superficies conformales. Se produce una presión de signo positivo en el sistema debido a la formación de una cuña entre dos superficies convergentes. La magnitud de la presión desarrollada no es bastante grande como para producir una deformación elástica de las superficies. Además, la película de lubricante que se forma es bastante gruesa como para impedir el contacto entre las dos superficies (superior a $1 \mu \mathrm{m})$.

- Lubricación elastohidrodinámica: es una forma de lubricación hidrodinámica donde la deformación elástica de las superficies se hace significativa. Los mismos puntos que son importantes en la lubricación hidrodinámica, lo son aquí también. Generalmente, la lubricación de régimen elastohidrodinámico se asocia con superficies no conformales.

- lubricación peculiar: en este régimen de lubricación, el grueso de fluido lubricante es bastante pequeño como para que las superficies lleguen al contacto y los efectos de la capa de fluido son insignificantes. En este régimen, el mecanismo de lubricación es gobernado por las propiedades físicas y químicas de las películas de lubricante de proporciones moleculares.
En el caso de la articulación natural, el tipo de régimen de lubricación que tiene es de régimen elastohidrodinámico, debido al bajo módulo de elasticidad y a la poca dureza de los materiales que la componen. Sin embargo, en el caso de la articulación de cadera de una prótesis artificial es diferente. Los materiales que la componen tiene un módulo de elasticidad más elevado que los de la articulación natural, y además, las superficies son altamente conformales $^{2-6}$.

Con estas características, el tipo de régimen de lubricación que más probablemente aparecerá en esta articulación es una lubricación hidrodinámica. Esta es la premisa que se supone al resto del trabajo.

\section{Ecuaciones de Navier-Stokes}

Las ecuaciones de Navier-Stokes, son las ecuaciones fundamentales de la ciencia del movimiento de fluidos. Se trata de ecuaciones en derivadas parciales, que si bien permiten describir el movimiento de los fluidos, presentan dificultades casi insalvables para poder obtener soluciones analíticas.

\section{Ecuación de Reynolds}

En el caso de los regímenes de lubricación, el año 1886 se presentó un conjunto de ecuaciones derivadas de las ecuaciones de Navier-Stokes por parte de $\mathrm{O}$. Reynolds. La ecuación así obtenida, también denominada ecuación de Reynolds, es la siguiente:

$$
\begin{array}{r}
0=\frac{\partial}{\partial x}\left(-\frac{p h 3}{12 \eta} \frac{\partial p}{\partial x}\right)+\frac{\partial}{\partial y}\left(-\frac{p h^{3}}{12 \eta} \frac{\partial p}{\partial y}\right)+\frac{\partial}{\partial x}\left[\frac{p h\left(u_{a}+u_{b}\right)}{2}\right] \\
+\frac{\partial}{\partial y}\left[\frac{p h\left(v_{a}+v_{b}\right)}{2}\right]+p\left(w_{a}-w_{b}\right)-p u_{a} \frac{\partial h}{\partial x}-p v_{a} \frac{\partial h}{\partial y}+h \frac{\partial p}{\partial t}
\end{array}
$$

Esta es la forma general de la ecuación de Reynols. Ahora bien, si nada más se considera un movimiento tangencial, donde:

$$
w_{a}=u_{a} \frac{\partial h}{\partial x}+v_{a} \frac{\partial h}{\partial y} ; w_{b}=0
$$

entonces la ecuación (2.1) será:

$$
\frac{\partial}{\partial x}\left(\frac{p h^{3}}{n} \frac{\partial p}{\partial x}\right)+\frac{\partial}{\partial y}\left(\frac{p h^{3}}{n} \frac{\partial p}{\partial y}\right)=12 \widetilde{u} \frac{\partial(p h)}{\partial x}+12 \widetilde{v} \frac{\partial(p h)}{\partial y}
$$


donde:

$$
\widetilde{\mathrm{u}}=\frac{\mathrm{u}_{\mathrm{a}}+\mathrm{u}_{\mathrm{b}}}{2}=\text { cte. } \quad \widetilde{\mathrm{v}}=\frac{\mathrm{v}_{\mathrm{a}}+\mathrm{v}_{\mathrm{b}}}{2}=\text { cte. }
$$$$
\frac{\mathrm{dp}}{\mathrm{dx}}=12 \widetilde{\mathrm{u}} \eta \frac{\mathrm{ph}-\mathrm{p}_{\mathrm{m}} \mathrm{h}_{\mathrm{m}}}{\mathrm{ph}_{3}}
$$

La ecuación (2.2) es aplicable tanto a lubricación de régimen elastohidrodinámico como a la de régimen hidrodinámico.

Como en la lubricación hidrodinámica las propiedades del fluido no varían de forma significativa en toda la capa, estas se pueden considerar constantes. Además por este régimen el movimiento es puramente de deslizamiento, de forma que $\mathrm{v}=0$. Por tanto, aplicando estas simplificaciones, la ecuación de Reynolds correspondiente es:

$$
\frac{\partial}{\partial x}\left(h^{3} \frac{\partial p}{\partial x}\right)+\frac{\partial}{\partial y}\left(h^{3} \frac{\partial p}{\partial y}\right)=12 \widetilde{u} \eta_{0} \frac{\partial h}{\partial x}
$$

Esta ecuación presenta el problema de ser de difícil resolución analítica. Se puede hacer una simplificación más, y considerar que la huida lateral de fluido es despreciable. Con esta simplificación, se puede llegar a obtener soluciones analíticas. La ecuación (2.3) se simplifica y queda:

$$
\frac{\partial}{\partial x}\left(\frac{p h^{3}}{\eta} \frac{\partial p}{\partial x}\right)=12 \widetilde{u} \frac{\partial(p h)}{\partial x}
$$

Si se integra la ecuación (2.4) respecto de x se obtiene:

$$
\frac{1}{\eta} \frac{\partial \mathrm{p}}{\partial \mathrm{x}}=\frac{12 \tilde{\mathrm{u}}}{\mathrm{h}^{2}}+\frac{\tilde{\mathrm{A}}}{\mathrm{ph}^{3}}
$$

Haciendo uso de las siguientes condiciones de contorno:

$$
\mathrm{dp} / \mathrm{dx}=0 ; \quad \text { donde } \mathrm{x}=\mathrm{x}_{\mathrm{m}} ; \rho=\rho_{\mathrm{m}} ; \quad \mathrm{h}=\mathrm{h}_{\mathrm{m}}
$$

se obtiene:

$$
A=-12 \hat{u} \rho_{m} h_{m}
$$

Sustituyendo en la ecuación (2.5) se obtiene:
La ecuación (2.6) es la forma integrada de la ecuación de Reynolds. El subíndice m, se refiere a la condición de los puntos donde $\mathrm{dp} / \mathrm{dx}=0$, eso es, como los puntos de presión máxima.

Si la viscosidad del fluido se considera constante a toda la capa del lubricante, la ecuación (2.6), se puede simplificar hasta obtener

$$
\frac{\mathrm{dp}}{\mathrm{dx}}=12 \tilde{\mathrm{u}} \eta \frac{\mathrm{h}-\mathrm{h}_{\mathrm{m}}}{\mathrm{h}^{3}}
$$

y considerando que ua $=0$, queda:

$$
\frac{d p}{d x}=6 u \eta \frac{h-h_{m}}{h^{3}}
$$

\section{Aplicación de la ecuación de Reynolds a un rodamiento axial infinito}

Para un rodamiento axial infinito la presión en la dirección axial se supone constante. Esta aproximación es válida para relaciones de diámetro/anchura inferiores a 0,5 .

Si se tiene en cuenta que $\mathrm{u}=\mathrm{r} \cdot \omega$, la ecuación (2.8) se convierte en:

$$
\frac{d p}{d x}=\frac{6 \eta_{o} r_{b} \omega_{b}\left(h-h_{m}\right)}{h^{3}}
$$

donde: $h_{m}$ es el grosor de la película cuando $\mathrm{dp} / \mathrm{dx}=0$.

Esta ecuación es válida, se si considera una viscosidad constante, esto es, que la presión no llegue a afectar la viscosidad del fluido lubricante.

Según la figura 1, si $d x=r_{b} d \varnothing$, la ecuación (2.9) queda:

$$
\frac{d p}{d \varnothing}=\frac{6 \eta_{\mathrm{o}} \mathrm{r}^{2} \mathrm{~b}_{\mathrm{b}}\left(\mathrm{h}-\mathrm{h}_{\mathrm{m}}\right)}{\mathrm{h}^{3}}
$$




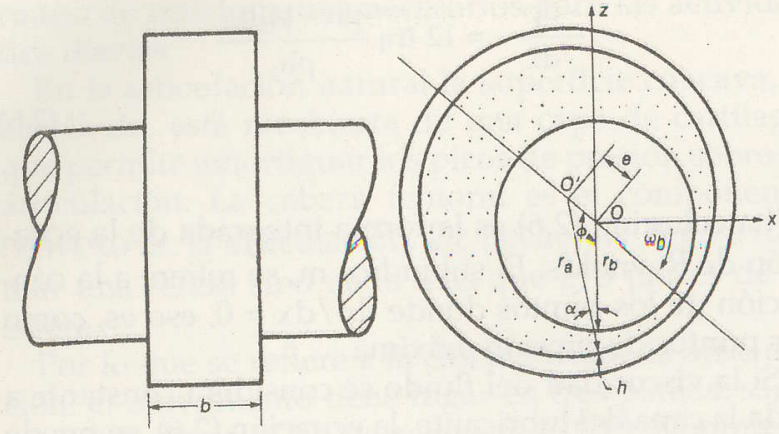

FIG. 1.-Esquema de un rodamiento axial infinito.

Este cambio de geometría es aceptable, ya que el grosor de la capa de lubricante, es relativamente pequeño respecto al radio y a la curvatura del rodamiento. Así, el film de lubricación se puede estudiar como una superficie lisa con una onda estacionaria de longitud de onda $2 \pi r_{b}$.

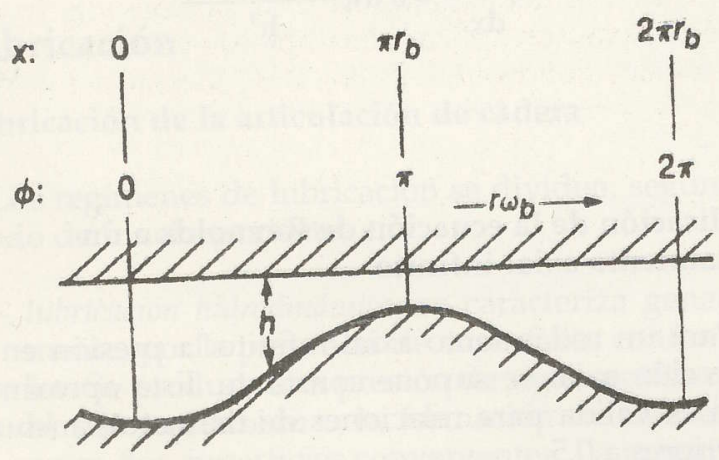

FIG. 2.-Esquema de la película de lubricación.

Si se desarrolla en serie y desestimamos los términos de orden superior, nos queda la siguiente expresión para el grueso h de la capa de lubricante:

$$
\mathrm{h}=\mathrm{c}(1+\varepsilon \cos \varnothing)
$$

donde $\varepsilon=\mathrm{e} / \mathrm{c}$

Sustituyendo esta expresión en la ecuación (2.10), queda:

$$
\frac{d p}{d \varnothing}=6 \eta_{\mathrm{o}} \omega_{\mathrm{b}}\left(\frac{\mathrm{r}_{\mathrm{b}}}{\mathrm{c}}\right)\left[\frac{1}{(1+\varepsilon \cos \varnothing)^{2}}-\frac{\mathrm{h}_{\mathrm{m}}}{\mathrm{c}(1+\varepsilon \cos \varnothing)^{3}}\right]
$$

de donde se puede obtener por integración una expresión de la distribución de presiones:

$$
p=6 \eta_{0} \omega_{b}\left(\frac{r_{b}}{c}\right) \int\left[\frac{1}{(1+\varepsilon \cos \varnothing)^{2}}-\frac{h_{\mathrm{min}}}{c(1+\varepsilon \cos \varnothing)^{3}}\right] d \varnothing+\widetilde{A}
$$

La ecuación (2.13) se integra de forma ordinaria con el cambio de variable $\gamma=\operatorname{tg}(\varnothing / 2)$. Desgraciadamente, en estos casos el uso de estos cambios de variable complican posteriores cálculos con esta ecuación.

Con el fin de evitar este problema, se introduce el siguiente cambio de variable:

$$
1+\varepsilon \cos \varnothing=\frac{1-\varepsilon^{2}}{1-\varepsilon \cos \gamma}
$$

conocido como la solución de Sommerfield. Haciendo uso de este cambio de variable y de condiciones de contorno periódicas, se obtiene la siguiente expresión:

$$
p-p_{o}=\frac{6 \eta_{o} \omega_{b}\left(r_{b} / c\right)^{2} \varepsilon \sin \varnothing(2+\varepsilon \cos \varnothing)}{\left(2+\varepsilon^{2}\right)(1+\varepsilon \cos \varnothing)^{2}}
$$

donde $p_{0}$ es la presión al punto de mínimo grosor de la capa de fluido.

\section{Aplicación de la ecuación de Reynolds a un rodamiento axial finito}

En el caso de un rodamiento de ancho finito, las fugas laterales no se pueden menospreciar y se ha de considerar su efecto a la capacidad de carga del rodamiento. Una aproximación analítica para este caso, se presentó a mediados de siglo. Esta solución supone que:

$$
u_{a}=v_{a}=v_{b}=0, \quad \partial x=r_{b} \partial \varnothing, \quad u_{b}=r_{b} \omega_{b}, \mu o=\text { cte. }
$$

Con estas suposiciones, se puede verificar que el término de presión inducida en la dirección circunferencial de la ecuación de Reynolds es pequeña frente 
al término de Couette. Entonces, la ecuación de Reynolds (2.3) queda reducida a:

$$
\frac{\partial}{\partial y}\left(h^{3} \frac{\partial p}{\partial y}\right)=6 \eta_{o} \omega_{b} \frac{\partial h}{\partial \varnothing}
$$

Esta ecuación es válida para rodamientos con un cociente diámetro/anchura $>2$.

\section{Aplicación de la ecuación de Reynolds para la articulación de cadera}

En el caso de la articulación de cadera, se puede asimilar su sistema de rodamiento de bola-acetábulo a un rodamiento que, en lugar de ser axial, presente curvatura en las dos direcciones. Entonces, habría que cambiar la ecuación que define $h$, que, considerando que la película de lubricación es bastante pequeña frente al radio de la bola, sería

$$
\mathrm{h}=\mathrm{c}[1+\varepsilon \cos \varnothing \cos \theta]
$$

Con esta ecuación se incorpora el hecho de que se trata de una superficie esférica. El ángulo $\varnothing$ representa una dirección de giro, y el ángulo $\theta$ representa la dirección de giro perpendicular al anterior.

Se considera que las condiciones que permiten llegar a la ecuación para el rodamiento axial finito se mantienen, de forma que se puede desarrollar para la articulación de cadera a partir de la ecuación (2.16).

En este caso, como h es dependiente de los ángulos $\theta$ y $\varnothing$, habrá que incluir la ecuación (2.17) dentro de la ecuación (2.16) teniendo en cuenta los cambios de variable que se producen por el hecho de pasar de una expresión esférica a una lineal. Entonces se tiene:

$$
\frac{\partial h}{\partial \varnothing}=-c \varepsilon \sin \varnothing \cos \theta
$$

y teniendo en cuenta que:

$$
d y=r_{b} d \theta
$$

la ecuación (2.16) queda:

$$
h^{3} \frac{\partial p}{\partial y}=-6 c \varepsilon \eta_{0} \omega_{b} r_{b} \sin \varnothing \sin \theta+\AA
$$

Agrupando las constantes, resulta:

$$
h^{3} \frac{\partial p}{\partial y}=c t e s \sin \varnothing \sin \theta+\tilde{A}
$$

donde ctes $=-6 \mathrm{c} \eta_{\mathrm{o}} \omega_{\mathrm{b}} \mathrm{r}_{\mathrm{b}} \varepsilon$

pasando $h$ a la derecha de la ecuación y haciendo el cambio de variable de $y$ por $\theta$, resulta:

$$
\frac{\partial \mathrm{p}}{\partial \theta}=\frac{\operatorname{ctes} \sin \varnothing \sin \theta}{(1+\varepsilon \cos \varnothing \sin \theta)^{3}}+\mathrm{A}^{\prime}
$$

dónde ctes $=-6 \eta_{\mathrm{o}} \omega_{\mathrm{b}} \mathrm{r}_{\mathrm{b}}{ }^{2} \varepsilon / \mathrm{c}^{2}$

La ecuación con derivadas parciales que resulta es de difícil solución analítica. Una posible vía de solución se basa en aplicar el cambio de variable de Sommerfield, siendo eps $=\varepsilon \cdot \cos \varnothing$. Si se hace así, la ecuación que resulta es:

$$
\frac{\partial p}{\partial 1}=\frac{\operatorname{ctes} \sin 1(1-\varepsilon \cos 1)^{2}}{\left(1+\varepsilon^{2}\right)^{2,5}}+A^{\prime \prime}
$$

La integración de la ecuación (2.23) resulta ser:

$$
p=\frac{\left(-\cos 1+\frac{1}{2} \operatorname{eps}(\cos 1)^{2}\right) \mathrm{ctes}}{\left(1-\mathrm{eps}^{2}\right)^{2}}+\mathrm{A}^{\prime \prime} 1+\mathrm{B}
$$

Si se hace uso de las condiciones de contorno para encontrar las constantes de integración $A$ y $B$, se obtienen las siguientes expresiones:

$$
\text { para: } p=0 \text { cuándo } \theta= \pm \pi / 2
$$

se obtiene que $A^{\prime \prime}=0$, y que $B$ es:

$$
B=-A \arccos (e p s)-\frac{1}{2} \frac{\text { eps }\left(-2+e^{2} s^{2}\right) \text { ctes }}{\left(-1+e^{2}\right)^{2}}
$$

Si se deshace el cambio de variable efectuado para 
hacer la integral, y se introduce el valor de B calculado a partir de las condiciones de contorno, y que la expresión de $\mathrm{p}$ es:

$$
\frac{\left(\frac{\cos (\mathrm{ro})-\mathrm{eps}}{1+\mathrm{eps} \cos (\mathrm{ro})}+\frac{1}{2} \frac{\text { eps }(-\cos (\mathrm{ro})-\mathrm{eps})^{2}}{(1+\mathrm{eps} \cos (\mathrm{ro}))^{2}}\right) \operatorname{ctes}}{\left(1-\mathrm{eps}^{2}\right)^{2}}-\frac{1}{2} \frac{\operatorname{eps}(-2+\text { eps })^{2} \text { ctes }}{\left(-1+\mathrm{eps}^{2}\right)^{2}}
$$

y deshaciendo los cambios de variables realizados, queda la expresión integrada de la presión en un punto para $0<\varnothing$ (d) $<\pi$ i $-\pi / 2<\theta$ (ro) $<\pi / 2$ :

$-6 \frac{\left(\frac{-\cos \left(r_{0}\right)-e p s \cos (d)}{1+e p s \cos (d) \cos \left(r_{0}\right)}+\frac{1}{2} \frac{e p s \cos (d)\left(-\cos \left(r_{0}\right)-e p s \cos (d)\right)^{2}}{\left(1+e p s \cos (d) \cos \left(r_{0}\right)\right)^{2}}\right) n 0 w_{b r b} \mathrm{rb}^{2} \operatorname{eps} \sin (d)}{\left(1-e p s^{2} \cos (d)^{2}\right)^{2} c^{2}}$

$$
+3 \frac{\operatorname{eps}^{2} \cos (d)\left(-2+e p s^{2} \cos (d)^{2}\right) n o w b \mathrm{rb}^{2} \sin (d)}{c^{2}\left(-1+e p s^{2} \cos (d)^{2}\right)^{2}}
$$

siendo:

d: $\varnothing$ (primera dirección angular)

ro: $\theta$ (segunda dirección angular)

eps: $\varepsilon(=\mathrm{e} / \mathrm{c})$

c: c (juego de la articulación)

no: $\eta_{0}$ (viscosidad absoluta del fluido intersticial)

wb: $\omega_{\mathrm{b}}$ (velocidad angular de giro)

rb: $r_{b}$ (radio de la bola de la articulación)

\section{Aplicación de la expresión de la presión a la articulación de la prótesis de cadera}

A partir de valores geométricos usuales en las prótesis de cadera empleadas actualmente y de una situación de carga extrema (salto), se hace el cálculo de presiones para una situación de carga dada.

Datos geométricos de la prótesis:

- radio de la bola: $11 \mathrm{~mm}=11.10^{-3} \mathrm{~m}$

- tolerancia del mecanizado: $10 \mu \mathrm{m}=1.10^{-5} \mathrm{~m}$.

- $\varepsilon$ se supone $=0.55$.

Datos de viscosidad del fluido intersticial:

$$
-\eta_{\mathrm{o}}-2 \cdot 10^{-3} \mathrm{~Pa}
$$

Datos cinemáticos:

$$
-\omega_{b}-10 s^{-1}
$$

A partir de estos valores, se puede calcular una gráfica de distribución de presiones del acetábulo de la prótesis:
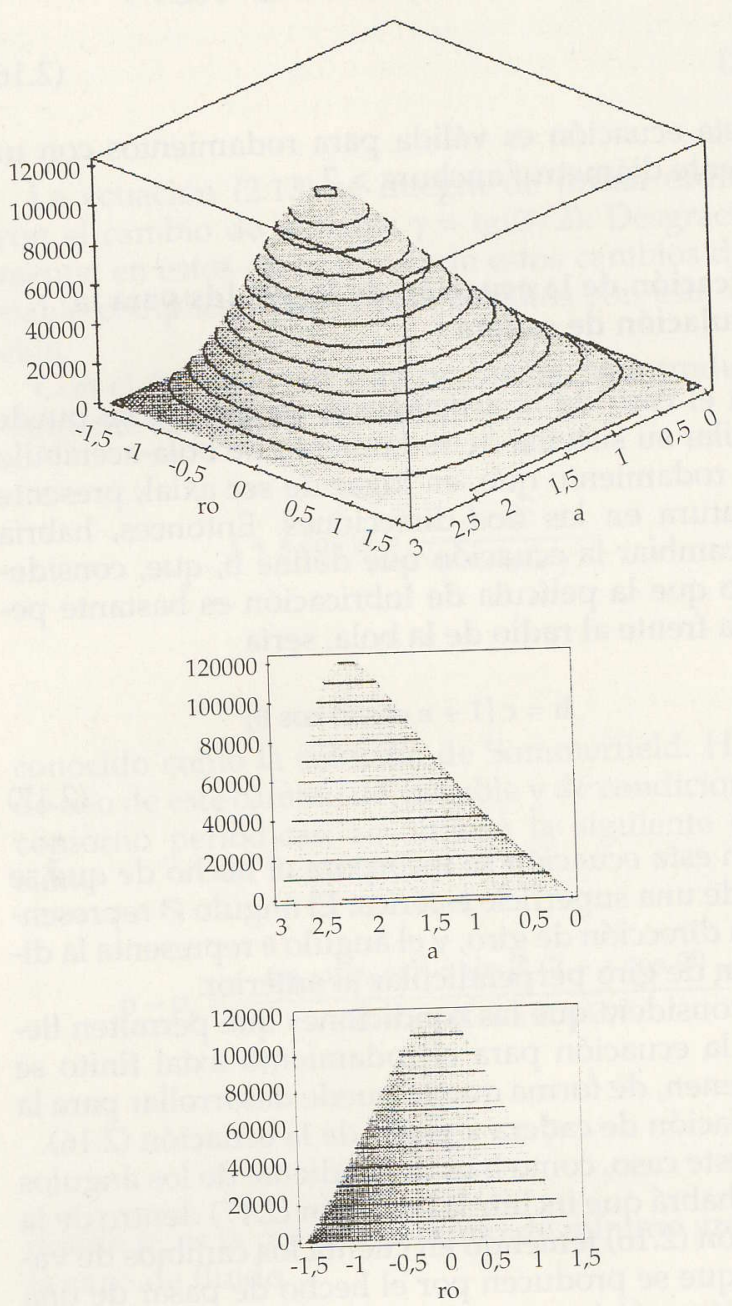

Se puede observar que se conserva una distribución simétrica respecto a la segunda dirección de movimiento, tal y como era de esperar.

\section{Discusión y conclusiones}

El punto de máxima presión se puede calcular haciendo la derivada de la función de la presión. El valor que se obtiene es:

$$
\begin{gathered}
\varnothing=2.37 \mathrm{rad} \\
\mathrm{P}_{\max }=121.8 \mathrm{KPa}
\end{gathered}
$$

Si se compara el valor máximo de presión obtenido con los valores de resistencia a la fatiga para el polie- 
tileno de ultra-alta densidad (UHMWPE), que es el material con que habitualmente se hacen los acetábulos, se puede comprobar que la tensión máxima de vida a fatiga aproximada para este material, de entre 10 y $20 \mathrm{MPa}$, es muy superior.

Ahora bien, los resultados obtenidos, se basan en datos que, si bien aproximados, no son del todo precisos. También se ha de tener en cuenta el hecho de que se ha empleado un punto de partida, la ecuación de Reynolds, aproximativo. Todo esto hay que tenerlo en cuenta, a fin de relativizar la exactitud de los valores obtenidos.

\section{Claves de los símbolos empleados}

A,B: constantes de integración

c: juego de la articulación

e: excentricidad de la bola respecto al centro geo-

métrico de la articulación

h: altura de la capa de fluido (ver Fig. 1)

p: presión en un punto

$\mathrm{r}_{\mathrm{b}}$ : radio de la bola de la articulación

t: tiempo

$\mathrm{u}, \mathrm{v}, \mathrm{w}$ : componentes del desplazamiento en las direcciones $\mathrm{x}, \mathrm{y}, \mathrm{z}$

$\varepsilon$ (eps): e/c $\varnothing, \theta$ : direcciones angulares perpendiculares de giro
de la articulación

$\gamma$ (1): variable de Sommerfield (tomada para la solución de integrales)

$\eta$ : coeficiente de viscosidad del fluido

$\eta_{0}$ : coeficiente de viscosidad absoluta del fluido $\rho$ : densidad del fluido

$\omega_{\mathrm{b}}$ : velocidad angular de giro de la bola en la articulación

\section{Bibliografía}

1. Collier JP, Mayor M, Williams IR, Surprenant BA, Currier B The Tradeoffs associated with modular hip prostheses. Clinical Orthopaedics and Related Research. 1995;311:91-101.

2. Hamrock BJ. Fundamentals of Fluid Film Lubrication. Graw Hill. London. 1995;218-45.

3. Srivastava VP. Two phase model of blood flow through stenosed tubes in the presence of a peripheral layer appligh stenoBiomechanics. 1996; vol.29(10):1377-82.

4. Tu C Deville M. Pulsatile 10 w of non-

roug 908

5. Anseth KS, Bowman N, Branon-Peppas L. Mechanical propermaterials, 1996:17:1647-7. experimental determination. Biomaterials. 1996;17:1647-57.

6. Fisher J, Dowson D. Tribology of total artificial joints. Proceedings of the Institution of Mechanical Engineers. Part H Journal of Engineering in Medicine. 1991; vol.205:75-89. 